\title{
Nomenclature and nosology for neuropathologic subtypes of frontotemporal lobar degeneration: an update
}

\author{
Ian R. A. Mackenzie • Manuela Neumann • Eileen H. Bigio • Nigel J. Cairns • Irina Alafuzoff • \\ Jillian Kril • Gabor G. Kovacs • Bernardino Ghetti · Glenda Halliday • Ida E. Holm • Paul G. Ince • \\ Wouter Kamphorst · Tamas Revesz • Annemieke J. M. Rozemuller • Samir Kumar-Singh • Haruhiko Akiyama • \\ Atik Baborie - Salvatore Spina · Dennis W. Dickson · John Q. Trojanowski · David M. A. Mann
}

Received: 9 November 2009/ Accepted: 10 November 2009/Published online: 19 November 2009

(C) The Author(s) 2009. This article is published with open access at Springerlink.com

One year ago, in this journal, we published a recommended nomenclature for the neuropathologic subtypes of frontotemporal lobar degeneration (FTLD) [7]. A major impetus behind this was to resolve the confusion that had arisen around the use of the term "FTLD with ubiquitinated inclusions" (FTLD-U), following the discovery that the molecular pathology of these cases was heterogeneous, with most, but not all, being characterized by pathological TDP-43 [6, 11]. In addition, a system of nosology was introduced that grouped the FTLD subtypes into broad

I. R. A. Mackenzie ( $\square)$

Department of Pathology and Laboratory Medicine,

Vancouver General Hospital and University of British Columbia, 855 West 12th Ave., Vancouver, BC V5Z 1M9, Canada

e-mail: ian.mackenzie@vch.ca

\section{Neumann}

Institute of Neuropathology, University Hospital of Zurich,

Zurich, Switzerland

E. H. Bigio

Department of Pathology, Northwestern University Feinberg

School of Medicine, Chicago, IL, USA

\section{N. J. Cairns}

Department of Pathology and Immunology,

Washington University School of Medicine, St. Louis, MO, USA

\section{Alafuzoff}

Department of Genetics and Pathology,

Uppsala University, Uppsala, Sweden

J. Kril

Department of Pathology, The University of Sydney,

Sydney, NSW, Australia

\section{G. G. Kovacs}

Institute of Neurology, Medical University of Vienna,

Vienna, Austria categories, based on the molecular defect that is most characteristic, according to current evidence. This system provided a concise and consistent terminology that has now been widely adopted in the literature. Another anticipated advantage was the ability to readily accommodate new discoveries. At the time, we did not anticipate how quickly this attribute would be put to use.

Although most FTLDs are characterized by cellular inclusion bodies composed of either tau (FTLD-tau) or TDP-43 (FTLD-TDP), approximately $10-15 \%$ of cases

\section{B. Ghetti $\cdot$ S. Spina}

Department of Pathology and Laboratory Medicine,

Indiana University School of Medicine, Indianapolis, IN, USA

G. Halliday

Prince of Wales Medical Research Institute,

University of New South Wales, Sydney, NSW, Australia

I. E. Holm

Laboratory for Experimental Neuropathology,

Danish Neuroscience Center, Aarhus University Hospital,

Aarhus, Denmark

P. G. Ince

Department of Neuroscience, University of Sheffield Medical School, Sheffield, UK

W. Kamphorst · A. J. M. Rozemuller

Department of Pathology, Vrije University Medical Centre, Amsterdam, The Netherlands

T. Revesz

Department of Molecular Neuroscience, UCL Institute of Neurology, Queen Square Brain Bank, Queen Square, London, UK

S. Kumar-Singh

VIB-Department of Molecular Genetics, University of Antwerp, Antwerp, Belgium 
remain, that include a number of uncommon FTLD subtypes, in which the pathologic protein is unknown. Recently, two studies identified mutations in the gene encoding the fused in sarcoma (FUS) protein (also known as translocated in liposarcoma, TLS), as the cause of familial amyotrophic lateral sclerosis (ALS) type 6 [5, 14]. The recognized clinical, genetic and pathological overlap between ALS and FTD, and the high degree of functional homology between FUS and TDP-43, prompted a number of subsequent studies that demonstrated that the inclusions of several of the tau/TDP-43-negative FTLDs are immunoreactive (ir) for FUS [8-10]. One such group are those cases with TDP-43-negative FTLD-U pathology, originally referred to as atypical FTLD-U (aFTLD-U) [6, 11]. According to the previous nomenclature recommendations, the neuropathology of these cases was designated as FTLD-UPS because the inclusions were only detectable with immunohistochemistry against proteins of the ubiquitin proteasome system (UPS) [7]. However, based on the discovery that all the ubiquitin-positive pathology in these cases is immunoreactive for FUS, we now recommend that they should be reclassified as FTLD-FUS [9]. In addition, the characteristic neuronal cytoplasmic inclusions of basophilic inclusions body disease (BIBD), previously of unknown biochemical composition, have also been shown to be consistently FUS-ir [8]. Perhaps most surprising has been the identification of abundant FUS-positive pathology in cases of neuronal intermediate filament inclusion disease (NIFID) [10]. The diagnostic criterion for NIFID is the presence of neuronal inclusions that are negative for tau, $\alpha$-synuclein and TDP-43 but immunoreactive for class IV intermediate filaments (IF) [1] and therefore the term FTLD-IF was designated in the previous nomenclature recommendations [7]. However, the finding that only a minority of the inclusions in NIFID are IF-ir, the absence of any identifiable genetic or molecular abnormality of IF

H. Akiyama

Tokyo Institute of Psychiatry, Tokyo, Japan

A. Baborie

Department of Neuropathology, The Walton Centre for

Neurology and Neurosurgery, Liverpool, UK

D. W. Dickson

Department of Neuropathology Laboratory, Mayo Clinic

College of Medicine, Jacksonville, FL, USA

J. Q. Trojanowski

Department of Pathology and Laboratory Medicine, University

of Pennsylvania School of Medicine, Philadelphia, PA, USA

D. M. A. Mann

Clinical Neuroscience Research Group, Greater Manchester

Neurosciences Centre, University of Manchester, Salford, UK in these cases and the recognition that immunohistochemistry for IF is not specific for this condition, is consistent with the possibility that another protein may be more central to the pathogenesis. The recent demonstration that a much larger proportion of the inclusions in NIFID are FUSir, that all the cells with IF-ir inclusions also contain pathological FUS, and that there are widespread FUS-ir glial inclusions, suggests that the abnormal accumulation of FUS may be more fundamental in the disease process and that IF pathology probably develops as a secondary process [10].

Taking these studies together, we now recommend that aFTLD-U, BIBD and NIFID should be grouped together under the designation of FTLD-FUS (Table 1). It is important to recognize, however, that this does not imply that a defect in FUS metabolism is known to be causal in any of these conditions. Rather, it simply indicates that they share FUS accumulation as the most prominent molecular pathology. Whether or not this indicates that aFTLD-U, BIBD and NIFID are actually all part of a continuous spectrum of disease must await detailed comparative clinicopathological studies of larger numbers of cases. Nonetheless, the presence of FUS pathology sets these cases apart and should aid in their neuropathological diagnosis and classification.

Although it now appears that most, if not all, cases of sporadic FTLD-UPS (i.e. aFTLD-U) have FUS-immunoreactive pathology [9], the designation FTLD-UPS remains appropriate for at least one condition: familial FTD linked to chromosome 3 (FTD-3), caused by mutations in the $C H M P 2 B$ gene. In addition to being negative for tau and TDP-43 [2], a recent study has shown that the ubiquitin/p62-immunoreactive neuronal inclusions in these cases do not label with antibodies against FUS [3]. Although these inclusions may eventually be discovered to contain a single major pathologic protein, it is also possible they have more heterogeneous composition that results from a primary defect of endosomal function [13]. Until this is determined, FTLD-UPS remains an appropriate designation for the neuropathology of FTD-3 and possibly for some FUS-negative sporadic cases.

With these recent advances, virtually all cases of FTLD can now be assigned to one of the three major molecular subgroups (FTLD-tau, FTLD-TDP or FTLD-FUS). This classification does not presuppose a primary role of the signature protein in pathogenesis (although in FTLD-tau and FTLD-TDP there is growing evidence to support this), but provides a logical way of grouping neuropathologic subtypes that is likely to have relevance regarding common disease mechanisms, diagnostic tests and possibly treatments. The specific role of the pathologic proteins and their relationship to causal gene defects is crucial information 
Table 1 Updated nomenclature for neuropathologic subtypes of frontotemporal lobar degeneration

\begin{tabular}{|c|c|c|c|c|}
\hline \multicolumn{2}{|l|}{2009 recommendation } & \multicolumn{2}{|l|}{2010 recommendation } & \multirow[t]{2}{*}{ Associated genes } \\
\hline Major molecular class & Recognized subtypes ${ }^{\mathrm{a}}$ & Major molecular class & Recognized subtypes ${ }^{\mathrm{a}}$ & \\
\hline FTLD-tau & $\begin{array}{l}\text { PiD } \\
\text { CBD } \\
\text { PSP } \\
\text { AGD } \\
\text { MSTD } \\
\text { NFT-dementia } \\
\text { WMT-GGI } \\
\text { Unclassifiable }\end{array}$ & FTLD-tau & $\begin{array}{l}\text { PiD } \\
\text { CBD } \\
\text { PSP } \\
\text { AGD } \\
\text { MSTD } \\
\text { NFT-dementia } \\
\text { WMT-GGI } \\
\text { Unclassifiable }\end{array}$ & $M A P T$ \\
\hline FTLD-TDP & $\begin{array}{l}\text { Types } 1-4 \\
\text { Unclassifiable }\end{array}$ & FTLD-TDP & $\begin{array}{l}\text { Types } 1-4 \\
\text { Unclassifiable }\end{array}$ & $\begin{array}{l}G R N \\
V C P \\
9 \mathrm{p} \\
(T A R D B P)^{\mathrm{b}}\end{array}$ \\
\hline FTLD-UPS & $\begin{array}{l}\text { FTD-3 } \\
\text { aFTLD-U }\end{array}$ & FTLD-UPS & FTD-3 & $C H M P 2 B$ \\
\hline $\begin{array}{l}\text { FTLD-IF } \\
\text { BIBD }\end{array}$ & NIFID & FTLD-FUS & $\begin{array}{l}\text { aFTLD-U } \\
\text { NIFID } \\
\text { BIBD }\end{array}$ & $(F U S)^{\mathrm{c}}$ \\
\hline FTLD-ni & & FTLD-ni & & \\
\hline
\end{tabular}

Entries in bold indicate major revisions

aFTLD-U, atypical frontotemporal lobar degeneration with ubiquitinated inclusions; AGD, argyrophilic grain disease; BIBD, basophilic inclusion body disease; $\mathrm{CBD}$, corticobasal degeneration; $\mathrm{CHMP} 2 \mathrm{~B}$, charged multivescicular body protein 2B; FTD-3, frontotemporal dementia linked to chromosome 3; FTLD, frontotemporal lobar degeneration; FUS, fused in sarcoma; GRN, progranulin gene; IF, intermediate filaments; MAPT, microtubule associated protein tau; MSTD, multiple system tauopathy with dementia; NFT-dementia, neurofibrillary tangle predominant dementia; ni, no inclusions; NIFID, neuronal intermediate filament inclusion disease; PiD, Pick's disease; PSP, progressive supranuclear palsy; TARDBP, transactive response DNA binding protein; TDP, TDP-43; UPS, ubiquitin proteasome system; VCP, valosin containing protein; WMT-GGI, white matter tauopathy with globular glial inclusions; 9p, genetic locus on chromosome 9p linked to familial amyotrophic lateral sclerosis and frontotemporal dementia

a Indicates the characteristic pattern of pathology, not the clinical syndrome. Note that FTDP-17 is not listed as a pathological subtype because cases with different MAPT mutations do not have a consistent pattern of pathology. These cases would all be FTLD-tau, but further subtyping would vary

${ }^{\mathrm{b}}$ Rare case reports of patients with clinical FTD and TDP-43 pathology associated with TARDBP genetic variants [4]

${ }^{c}$ One patient reported with a FUS mutation and FTD/ALS clinical phenotype but no description of pathology [12]

that requires further neuropathological and experimental investigations.

Open Access This article is distributed under the terms of the Creative Commons Attribution Noncommercial License which permits any noncommercial use, distribution, and reproduction in any medium, provided the original author(s) and source are credited.

\section{References}

1. Cairns NJ, Uryu K, Bigio E et al (2004) $\alpha$-Internexin in neuronal intermediate filament inclusion disease and other neurodegenerative diseases. Acta Neuropathol 108:213-223. doi:10.1007/ s00401-004-0882-7

2. Holm IE, Englund E, Mackenzie IRA, Johannsen P, Isaacs A (2007) A reassessment of the neuropathology of frontotemporal dementia linked to chromosome 3 (FTD-3). J Neuropathol Exp Neurol 66:884-891. doi:10.1097/nen.0b013e3181567f02
3. Holm IE, Isaacs A, Mackenzie IRA (2009) Absence of FUSimmunoreactive pathology in frontotemporal dementia linked to chromosome 3 (FTD-3) caused by mutation in the CHMP2B gene. Acta Neuropathol 118:719-720. doi:10.1007/s00401009-0593-1

4. Kovacs GG, Murrell JR, Horvath S et al (2009) TARDBP variation associated with frontotemporal dementia, supranuclear gaze palsy and chorea. Mov Disord 24:1843-1847. doi:10.1002/mds. 22697

5. Kwiatkowski TJ, Bosco DA, LeClerc AL et al (2009) Mutations in the FUS/TLS gene on chromosome 16 cause familial amyotrophic lateral sclerosis. Science 323:1205-1208. doi:10.1126/ science. 1166066

6. Mackenzie IRA, Foti D, Woulfe J, Hurwitz TA (2008) Atypical frontotemporal lobar degeneration with ubiquitin-positive, TDP43-negative neuronal inclusions. Brain 131:1282-1293. doi: 10.1093/brain/awn061

7. Mackenzie IR, Neumann M, Bigio EH et al (2009) Nomenclature for neuropathologic subtypes of frontotemporal lobar degeneration: consensus recommendations. Acta Neuropathol 117:15-18. doi:10.1007/s00401-008-0460-5 
8. Munoz DG, Neumann M, Kusaka H et al (2009) FUS pathology in basophilic inclusion body disease. Acta Neuropathol 118:617627. doi:10.1007/s00401-009-0598-9

9. Neumann M, Rademakers R, Roeber S, Baker M, Kretzschmar HA, Mackenzie IRA (2009) Frontotemporal lobar degeneration with FUS pathology. Brain 132:2922-2931. doi:10.1093/brain/ awp214

10. Neumann M, Roeber S, Kretzschmar HA, Rademakers R, Baker M, Mackenzie IRA (2009) Abundant FUS pathology in neuronal intermediate filament inclusion disease. Acta Neuropathol 118:605-616. doi:10.1007/s00401-009-0581-5

11. Roeber S, Mackenzie IR, Kretzschmar HA, Neumann M (2008) TDP-43-negative FTLD-U is a significant new clinico- pathological subtype of FTLD. Acta Neuropathol 116:147-157. doi:10.1007/s00401-008-0395-x

12. Ticozzi N, Silani V, LeClerc AL et al (2009) Analysis of FUS gene mutation in familial amyotrophic lateral sclerosis within an Italian cohort. Neurology 73:1180-1185. doi:10.1212/WNL. 0b013e3181bbff05

13. Urwin H, Ghazi-Noori S, Collinge J, Isaacs A (2009) The role of CHMP2B in frontotemporal dementia. Biochem Soc Trans 37:208-212. doi:10.1042/BST0370208

14. Vance C, Rogelj B, Hortobagyi T et al (2009) Mutations in FUS, an RNA processing protein, cause familial amyotrophic lateral sclerosis type 6. Science 323:1208-1211. doi:10.1126/science. 1165942 